\title{
A Novel Frequency Domain Iterative Image Registration Algorithm Based on Local Region Extraction
}

\author{
Xiujie Qu, Haili Huo, Sitong Lian, and Fei Zhao \\ School of Information and Electronics, Beijing Institute of Technology, Beijing 100081, China \\ Correspondence should be addressed to Xiujie Qu; quxiujie@bit.edu.cn
}

Received 31 July 2014; Revised 19 September 2014; Accepted 25 September 2014

Academic Editor: Wuhong Wang

Copyright (C) 2015 Xiujie Qu et al. This is an open access article distributed under the Creative Commons Attribution License, which permits unrestricted use, distribution, and reproduction in any medium, provided the original work is properly cited.

\begin{abstract}
Because of the differences of imaging time, position between sensor and target position, scaling, rotation, translation, and other transformations between the series of images will be generated by the imaging system. The conventional phase correlation algorithm has been widely applied because of its advantages of high speed, precision, and weak influence of the geometric distortion when computing these changing parameters. However, when the scaling factor and the rotation angle are too large, it is difficult to use the conventional phase correlation method for high precision registration. To solve this problem, this paper presents a novel method, which combines the speeded up robust features algorithm and the phase correlation method under the log polar. Through local region extraction and reusing a two-step iterative phase correlation algorithm, this method avoids excessive computation and the demand of characteristics of the image and effectively improves the accuracy of registration. A plurality of visible light image simulation verifies that this is a fast, accurate, and robust algorithm, even when the image has large angle rotation and large multiple scaling.
\end{abstract}

\section{Introduction}

Image registration is a process of matching and superposing two or more images obtained from different time, different angles, and different sensors [1]. With the rapid development of science and technology, image registration technique has been widely used in medical image processing, computer vision, remote sensing image processing, target recognition, and so forth. At home and abroad, three aspects are mainly concentrated in the research on image registration at present: feature space, search strategy, and similarity measure [24]. Image registration methods can be roughly divided into two categories: first, the spatial domain registration method relying on the gray information, for example, mutual information based registration and feature based registration [5]; second, the frequency domain registration method, for example, cross correlation registration and phase correlation registration based on Fourier transform [6-8]. The conventional spatial domain registration method has a large amount of calculation and slow execution speed. It is difficult to effectively suppress the image geometric distortion. For the image without distinguishing characteristic, feature based registration method also cannot reach the expected purpose. When the scaling factor and the rotation angle are too large, the conventional phase correlation method cannot get satisfying result of registration either. Literature [9] proposed an algorithm based on mutual information and FourierMellin, which can achieve the image registration with large scaling factor and the rotation angle at the same time. But it is difficult to meet the real-time registration because it is time consuming. Literature [10] proposed an algorithm which is of great randomness and is very difficult to guarantee to obtain suitable region when intercepting the reference image and the image to be registered. This paper proposes a novel frequency domain iterative image registration algorithm based on local region extraction. First, one pair of angular points which are close to the central region between the reference image and the image to be registered are detected, and the local area is intercepted at the same time by the SURF algorithm. Second, the large scaling factor of intercepted image and the rotation angle can be roughly estimated by the FourierMellin algorithm. Then, those parameters can be compensated according to the estimated results, precisely estimated by Fourier-Mellin algorithm once again. Finally, the precise 
registration parameters are obtained. The simulation results of multiple images indicate that this method has obvious advantages of speed and accuracy of image registration and shows good universality and robustness compared with the conventional phase correlation algorithm and the method of literature [9].

\section{Phase Correlation Registration Algorithm}

2.1. Phase Correlation Method for the Translation Parameters. Phase correlation method is proposed by Kuglin and Hines. First, the images are transformed from the time domain to the frequency domain through the fast Fourier transform. Then the translation vectors between the two images are directly calculated through their cross power spectrum, thus determining the relations of the image location [11, 12]. Phase correlation algorithm has now been extended to image registration applications in which the two images differ from each other in rotation and scale [13]. Rotation and scaling transformation on the Cartesian coordinates can be converted to translation transform on the log-polar coordinates, so the image rotation and scaling parameters can be extracted according to phase correlation algorithm. This paper discusses the case under the condition of relatively large scaling factor and the rotation angle. Principle of phase correlation technique: given two images $I_{1}$ and $I_{2}$, which differ only by a displacement $(d x, d y)$, the relationship of the two is expressed as follows:

$$
I_{2}(x, y)=I_{1}(x-d x, y-d y)
$$

Assuming the digital image is an $M \times N$ dimensional matrix, where $F_{1}$ and $F_{2}$ are, respectively, the Fourier transform for the two images, so they are expressed as follows:

$$
\begin{aligned}
& F_{1}\left(\omega_{x}, \omega_{y}\right)=\sum_{x, y} \frac{I_{1}(x, y)}{\sqrt{M N}} \exp \left[-2 \pi j\left(\frac{\omega_{x} x}{M}+\frac{\omega_{y} y}{N}\right)\right], \\
& F_{2}\left(\omega_{x}, \omega_{y}\right)=\sum_{x, y} \frac{I_{2}(x, y)}{\sqrt{M N}} \exp \left[-2 \pi j\left(\frac{\omega_{x} x}{M}+\frac{\omega_{y} y}{N}\right)\right] .
\end{aligned}
$$

$F_{1}, F_{2}$ can be related by

$$
F_{2}\left(\omega_{x}, \omega_{y}\right)=e^{-j\left(\omega_{x} d x+\omega_{y} d y\right)} F_{1}\left(\omega_{x}, \omega_{y}\right) .
$$

Through the formula above, two images which have the same Fourier magnitude but a phase difference which can be directly calculated by their cross power spectrum. The phase of the cross power spectrum is as follows:

$$
e^{-j\left(\omega_{x} d x+\omega_{y} d y\right)}=\frac{F_{1}\left(\omega_{x}, \omega_{y}\right) F_{2}^{*}\left(\omega_{x}, \omega_{y}\right)}{\left|F_{1}\left(\omega_{x}, \omega_{y}\right) F_{2}^{*}\left(\omega_{x}, \omega_{y}\right)\right|},
$$

where the asterisk ${ }^{*}$ is the complex conjugate of $F_{1}$ and $F_{2}$. The inverse Fourier transform of the phase difference is a delta function centered at the displacement. By searching the peak of the inverse Fourier transform, the maximum value of the function can be obtained, which in this case is the point of the registration.
2.2. Phase Correlation Algorithm on the Log-Polar Coordinates. Log-polar coordinate transformation expressed the changes of image description. The Cartesian coordinates system is shown in Figure 1 which represents the scene plane coordinates. Log-polar coordinates are the converted coordinates, as shown in Figure 2.

If the image in the $x, y$ directions is, respectively, multiplied by a scaling parameter, $(x, y)$ in the Cartesian coordinates will become $(a x, a y)$; then log transformation can be used to simplify the process:

$$
(\ln (a x), \ln (a y))=((\ln a+\ln x),(\ln a+\ln y)) .
$$

The scale transformation of the Cartesian coordinates system is corresponding to the translation transform of log-polar coordinates system $[14,15]$. Similarly, the angle transform in Cartesian coordinates system is also corresponding to their counterparts in log-polar coordinates system, which can simplify the registration process by transforming images into log-polar coordinates system when angle exists. If the image $I_{2}$ is a scaled, rotated, and translated version of $I_{1}$, then $I_{2}$ can be expressed as follows:

$$
\begin{aligned}
& I_{2}(x, y) \\
& =I_{1}\left(x a \cos \theta_{0}+y a \sin \theta_{0}+d x,-x a \sin \theta_{0}+y a \cos \theta_{0}+d y\right),
\end{aligned}
$$

where $\theta_{0}$ represents a rotation angle, $(d x, d y)$ represents the amount of shift between the images, and $\alpha$ represents a scaling parameter between images. First we apply the Fourier transform on both of the images and get the following formula:

$$
\begin{aligned}
& I_{2}\left(\omega_{x}, \omega_{y}\right) \\
& =e^{-2 \pi j\left(\omega_{x} d x+\omega_{y} d y\right)} \\
& \quad \times I_{1}\left(\omega_{x} a \cos \theta_{0}+\omega_{y} a \sin \theta_{0},-\omega_{x} a \sin \theta_{0}+\omega_{y} a \cos \theta_{0}\right) .
\end{aligned}
$$

Then the log-polar transform can be applied to the magnitude spectrum of the Fourier transform. The rotation and scale parameter are obtained by using the phase correlation on the log-polar image:

$$
\begin{array}{r}
M_{2}\left(\omega_{x}, \omega_{y}\right) \\
=M_{1}\left(\omega_{x} a \cos \theta_{0}+\omega_{y} a \sin \theta_{0},-\omega_{x} a \sin \theta_{0}+\omega_{y} a \cos \theta_{0}\right), \\
\text { where } M=|F| .
\end{array}
$$

If $\alpha$ is not equal to 1 , the registration of two images contains scaling transformation. By converting the magnitude spectra to the log-polar domain, as in (9), the scaling parameters can be easily extracted from (4):

$$
M_{2}(\gamma, \theta)=M_{1}\left(a \gamma, \theta-\theta_{0}\right)
$$




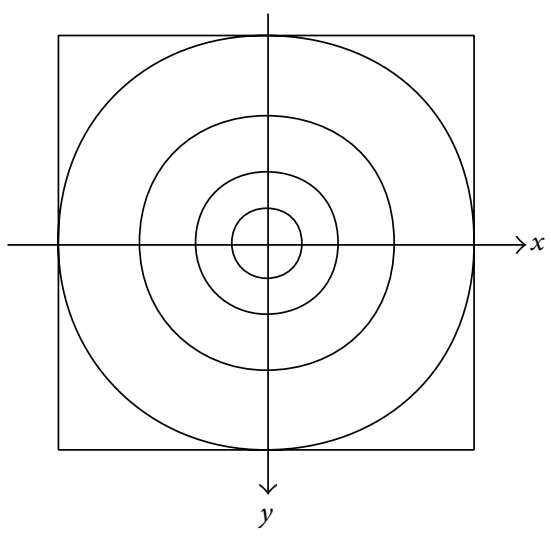

FIGURE 1: Cartesian coordinate.

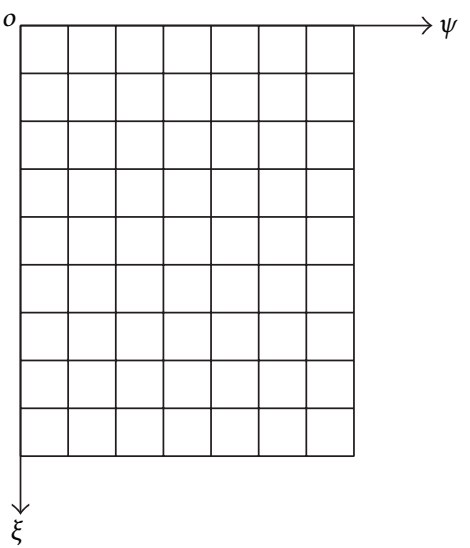

FIGURE 2: Log-polar coordinate.

The coordinate $\gamma$ can be transformed to the logarithmic coordinates:

$$
M_{2}(\lg \gamma, \theta)=M_{1}\left(\lg a+\lg \gamma, \theta-\theta_{0}\right) .
$$

Assuming $\omega=\lg \gamma, \eta=\lg \alpha$, the formula can be rewritten as follows:

$$
M_{2}(\omega, \theta)=M_{1}\left(\omega+\eta, \theta-\theta_{0}\right) .
$$

Then the phase correlation method can be used to compute the scaling parameter:

$$
a=e^{\eta} .
$$

\section{Phase Correlation Algorithm Based on SURF Feature Points}

When the scaling ratio and rotation angle of the image to be registered are too large compared to the original image, it is difficult for the conventional phase correlation method to compute the registration parameters accurately. If the local area with the same information in two images is extracted and the registration parameters are obtained using Fourier-Mellin algorithm, precision will be significantly improved. SURF is a method which has the advantages of high speed, stable scale invariant, and invariant rotation features and which is a fast and robust method of selecting feature points [16, 17]. In this paper, the local area with the same information in two images is extracted by using the SURF feature algorithm. Then by the Fourier-Mellin algorithm with two-step iterative from coarse to fine, the registration parameters can be obtained accurately. The specific steps are as follows.

(1) Construct the scale space of the reference image and the image to be registered and extract the feature points with scale invariant feature in the scale space using the Hessian matrix $t$.

(2) Confirm the main direction of the characteristic points and rotate the coordinate axis according to the main direction.

(3) Generate the 64-feature vector by describing the feature of feature points after synthesizing the spatial distribution information and determine the corresponding points of the reference image and the image to be registered.

(4) Take one pair of the same point near the image center point and intercept a small area of the same size which is about half of an image.

(5) Compute the intercepted image scaling factor and rotation angle roughly using the log-polar coordinate.

(6) Preliminary compensate the interception of images to be registered for rotation and scaling and compute the fine scaling factor and the rotation angle by the Fourier-Mellin algorithm again.

(7) Fuse the scaling factor and the rotation angle obtained from two-step iterative and compute and extract the center area of the two images according to the difference of images between the size, scaling factor, and rotation angle.

(8) Compute the translation parameters of the two images using the extracted image of the center region.

(9) Compensate the image to be registered by scaling, rotating, and translating and output the registration image.

\section{Experimental Results and Analysis}

As shown in Figure 3, two pieces of $512 * 512$ images are selected to validate the image registration algorithm proposed in this paper. The reference image is shown in Figure 3(a). After scaling 1.53 times, rotating 40.67 degrees and translating $(20,30)$ pixels, the image to be registered is obtained (Figure 3(b)). Figure 3(c) is the same point detected between the reference image and the image to be registered using the SURF algorithm. Figures 3(d) and 3(e) are the local areas intercepted from Figures $3(\mathrm{a})$ and $3(\mathrm{~b})$ according to Figure 3(c). Figures 3(f) and 3(g) are the images transformed from Figures 3(d) and 3(e) according to the $\log$ polar. Figure 3(h), which has a clear peak, is the threedimensional display obtained by the cross power spectrum 


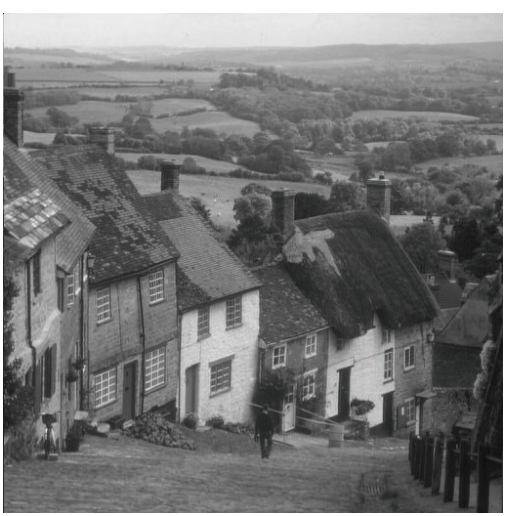

(a)

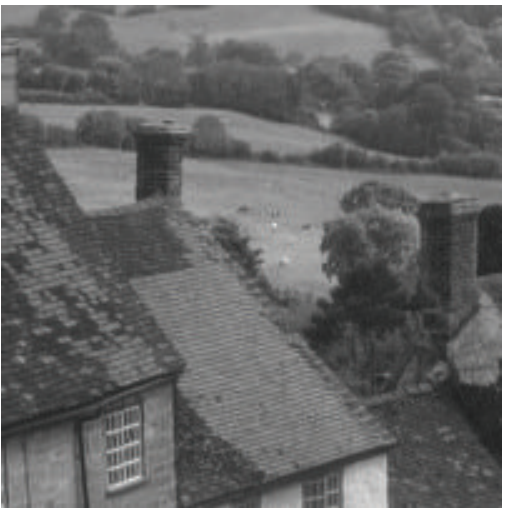

(d)

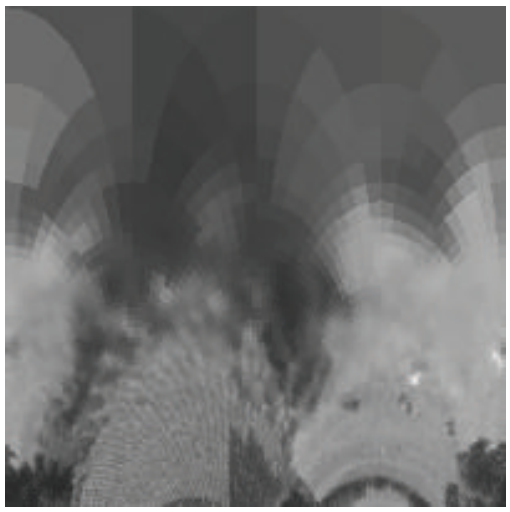

(g)

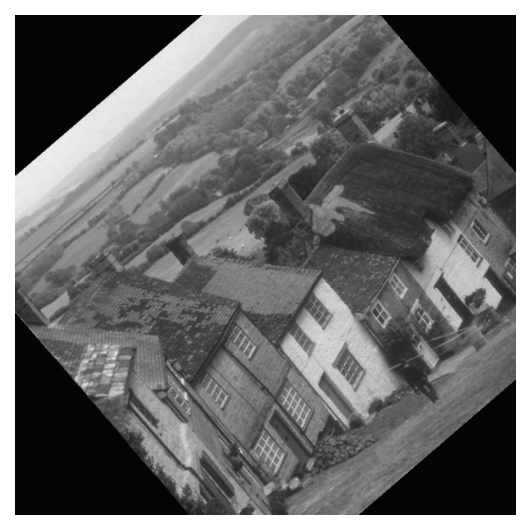

(b)

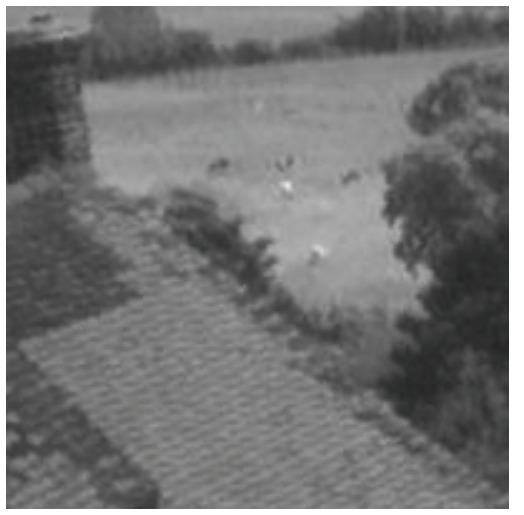

(e)

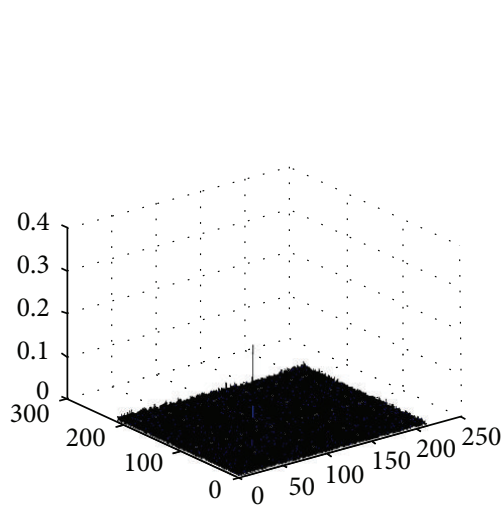

(h)

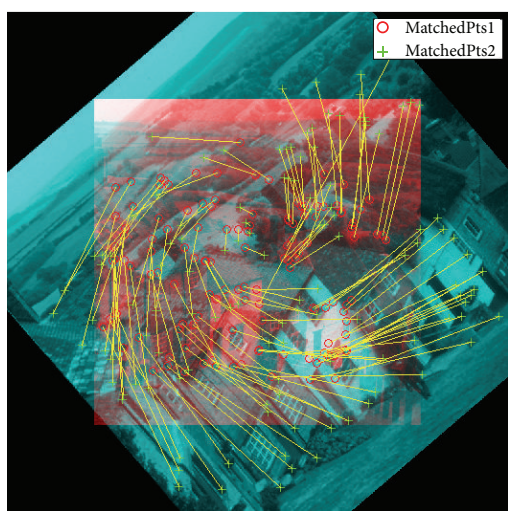

(c)

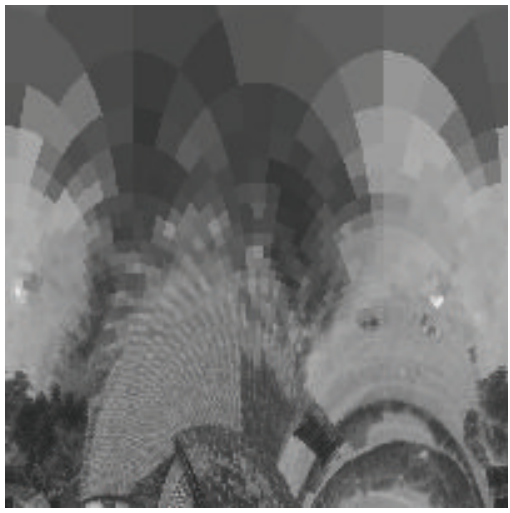

(f)

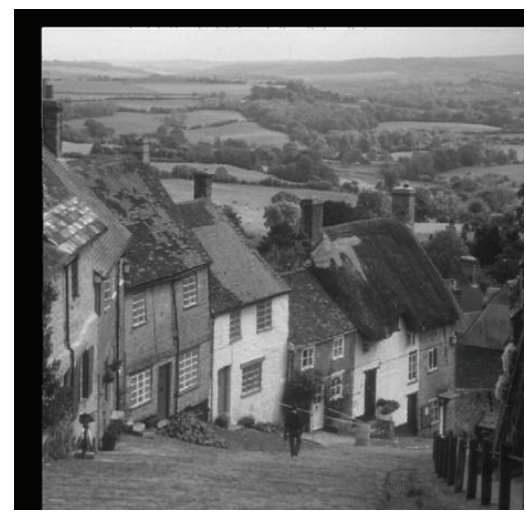

(i)

FIGURE 3: Using the proposed algorithm to calculate the translation, rotation, and scaling parameters.

after inversing Fourier transform. Figure 3(i) is the registered image.

As can be seen from Table 1, the estimation error of the translation parameters is within 1 pixel through the proposed algorithm. The estimation error of rotation angle is less than 0.5 degree, and the error estimates of scaling factor are less than 0.005. Compared with the conventional phase correlation algorithm, this algorithm improves the accuracy of registration without loss of speed.

It can be seen from Table 2, the two-step iterative algorithm proposed in this paper consumes some computation time, but the accuracy of registration will be more satisfactory.

Literature [9] presents an image registration method based on mutual information and Fourier-Mellin, which integrate the accuracy of calculating linear parameters in Fourier-Mellin-based method and the accuracy of calculating rotation and scale parameters in mutual information based method. Using this method can obtain precise registration parameters but takes a long time. Figure 4 verifies the algorithm proposed in this paper compared with literature [9]. The reference image is shown in Figure 4(a). After 


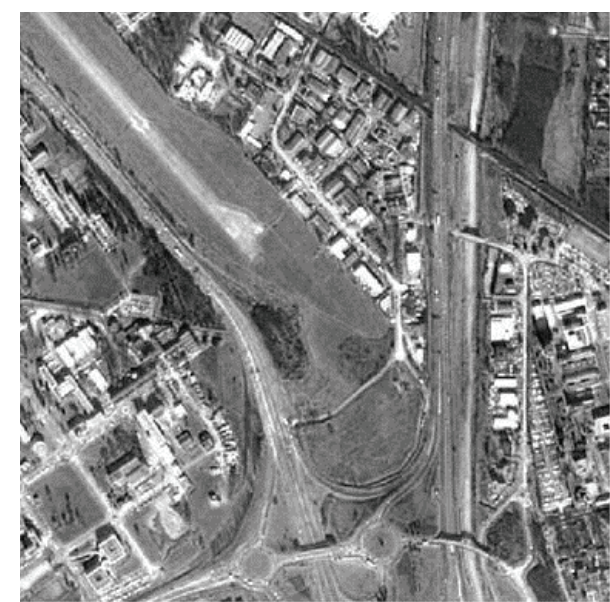

(a)

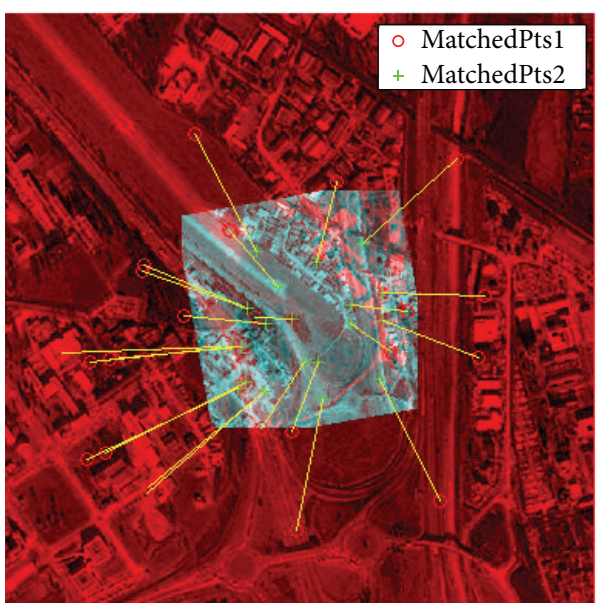

(c)

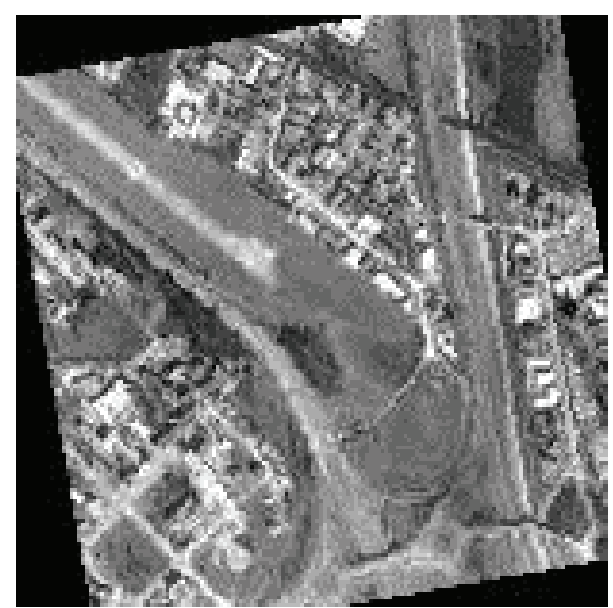

(b)

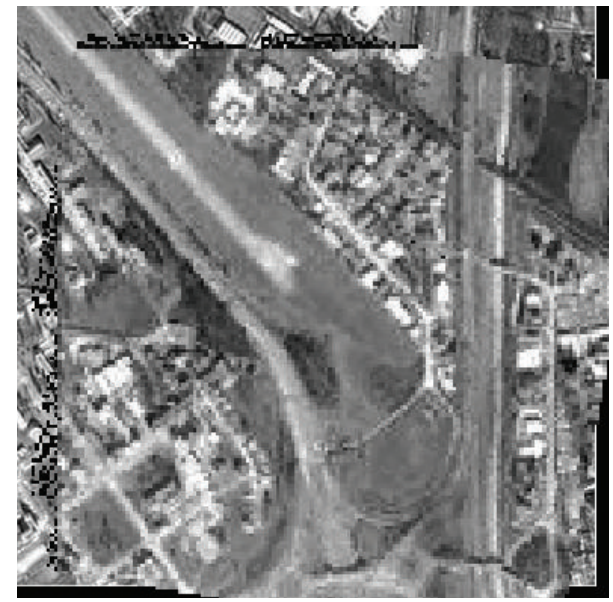

(d)

Figure 4: Remote sensing image registration results.

TABLE 1: Comparison of this algorithm and the traditional phase correlation algorithm.

\begin{tabular}{lccc}
\hline & $\begin{array}{c}\text { The known } \\
\text { parameters }\end{array}$ & $\begin{array}{c}\text { Calculated } \\
\text { parameters } \\
\text { by this } \\
\text { algorithm }\end{array}$ & $\begin{array}{c}\text { Calculated } \\
\text { parameters } \\
\text { by phase } \\
\text { correlation } \\
\text { algorithm }\end{array}$ \\
\hline The zoom & 1.53 & 1.5314 & 1.4978 \\
\hline Rotation angle & 40.67 & 40.67 & 40.4082 \\
\hline Translation vector & $(20,30)$ & $(19,30)$ & $(25,35)$ \\
\hline Registration time & & $2.290529 \mathrm{~s}$ & $3.0086 \mathrm{~s}$ \\
\hline
\end{tabular}

scaling 0.4 times, rotating 10 degrees, and translating (10, 18) pixels, the image to be registered is obtained shown in Figure 4(b). Figure 4(c) is the same point which is using the SURF algorithm. Figure 4(d) is the registered image. The registration results are shown in Table 3.

As can be seen from Table 3, the algorithm based on Fourier-Mellin and mutual information is at slow speed and
TABLE 2: The performance comparison results of before iteration and after iteration.

\begin{tabular}{lccc}
\hline & $\begin{array}{c}\text { The known } \\
\text { parameters }\end{array}$ & $\begin{array}{c}\text { Before } \\
\text { iteration }\end{array}$ & $\begin{array}{c}\text { After } \\
\text { iteration }\end{array}$ \\
\hline The zoom & 1.53 & 1.5693 & 1.5314 \\
\hline Rotation angle & 40.67 & 40.78 & 40.67 \\
\hline Translation vector & $(20,30)$ & $(19,29)$ & $(19,30)$ \\
\hline Registration time & & $2.06864 \mathrm{~s}$ & $2.290529 \mathrm{~s}$ \\
\hline
\end{tabular}

needs a large amount of calculation. It is difficult to find the perfect balance between registration accuracy and calculation speed, while the algorithm proposed in this paper can greatly improve the registration rate without loss of accuracy.

In order to verify the universality of this algorithm, these images are registered with different brightness and clarity, respectively. The registration in images with different clarity is shown in Figure 5. The reference image is a fuzzy image shown in Figure 5(a). After scaling 1.2 times, rotating 15 degrees, and translating $(5,15)$ pixels, the image to be 


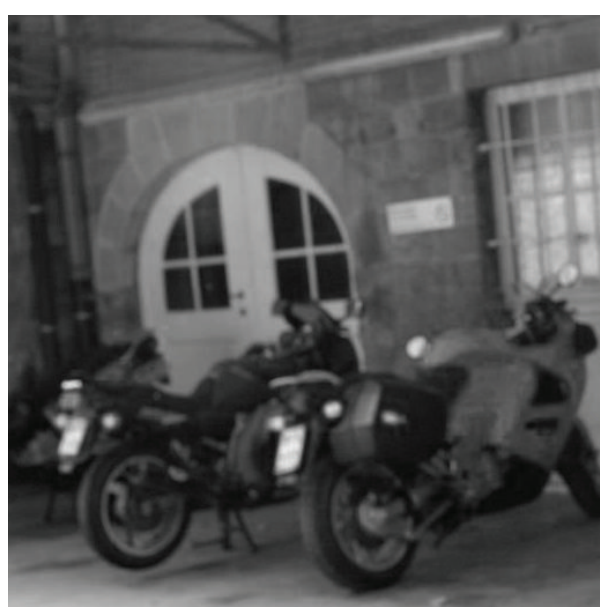

(a)

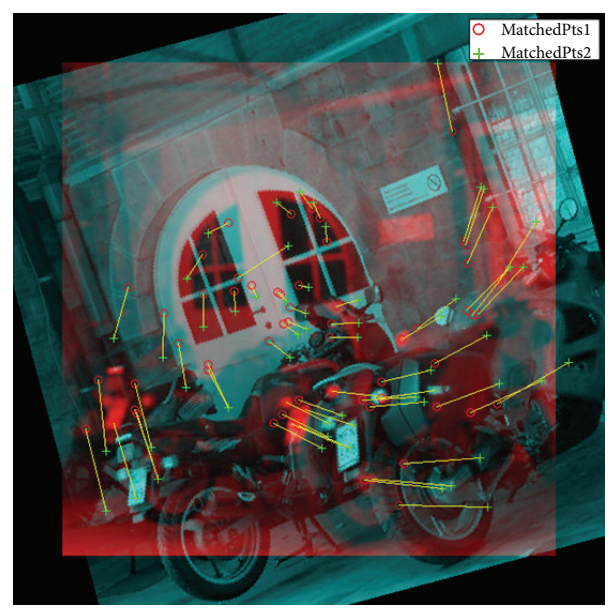

(c)

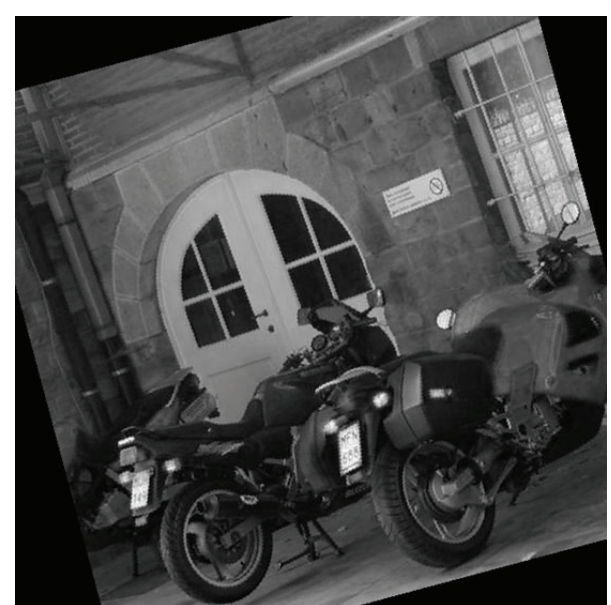

(b)

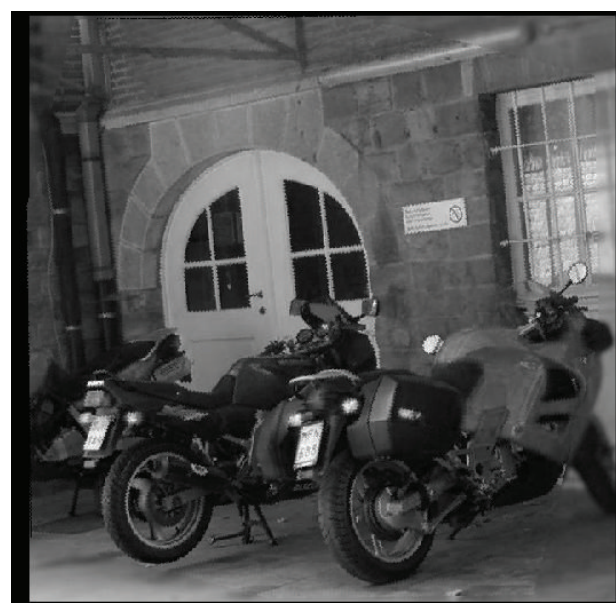

(d)

FIGURE 5: The images with different clarity to be registered.

TABLE 3: Comparison of this algorithm and the mutual information algorithm.

\begin{tabular}{lccc}
\hline & $\begin{array}{c}\text { The known } \\
\text { parameters }\end{array}$ & $\begin{array}{c}\text { Calculated } \\
\text { parameters } \\
\text { by this } \\
\text { algorithm }\end{array}$ & $\begin{array}{c}\text { Calculated } \\
\text { parameters } \\
\text { by mutual } \\
\text { information } \\
\text { algorithm }\end{array}$ \\
\hline The zoom & 0.4 & 0.4020 & 0.4005 \\
\hline Rotation angle & 10 & 10.000 & 9.9908 \\
\hline Translation vector & $(10,18)$ & $(10,18)$ & $(10,18)$ \\
\hline Registration time & & $1.78 \mathrm{~s}$ & $90.7843 \mathrm{~s}$ \\
\hline
\end{tabular}

registered is obtained which is shown in Figure 5(b). Figure 5(c) is the same point which is using the SURF algorithm. Figure $5(\mathrm{~d})$ is the registered image. The registration results are shown in Table 4 . The experimental results fully demonstrate the superiority of this method.

Then we register the images with different brightness. Figure 6(a) is the reference image with dark brightness. After
TABLE 4: Comparison of this algorithm and the known parameters.

\begin{tabular}{lcc}
\hline & $\begin{array}{c}\text { The known } \\
\text { parameters }\end{array}$ & $\begin{array}{c}\text { Calculated } \\
\text { parameters }\end{array}$ \\
\hline The zoom & 1.2 & 1.2015 \\
\hline Rotation angle & 15 & 14.7656 \\
\hline Translation vector & $(5,15)$ & $(5,16)$ \\
\hline Registration time & & $2.98 \mathrm{~s}$ \\
\hline
\end{tabular}

scaling 1.2 times, rotating 15 degrees, and translating $(5,15)$ pixels, the image to be registered is obtained which is shown in Figure 6(b). Figure 6(c) is the same point which is using the SURF algorithm. Figure $6(\mathrm{~d})$ is the registered image. The registration results are shown in Table 5.

\section{Conclusion}

Phase correlation method is a subpixel image registration technique which is widely used. The large scaling factor and 


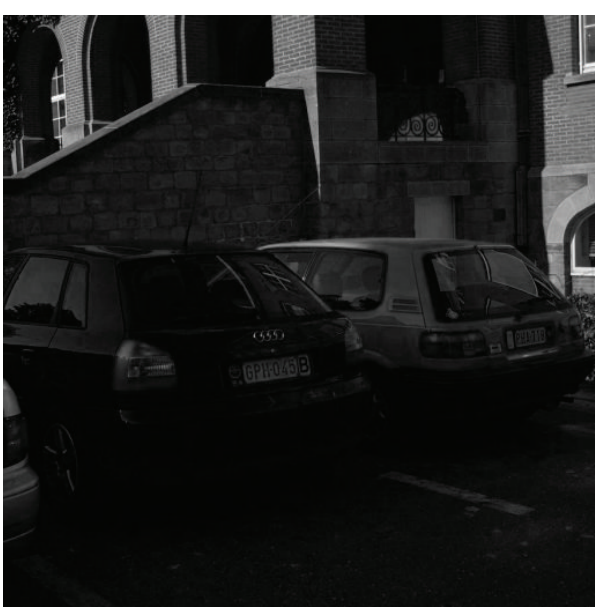

(a)

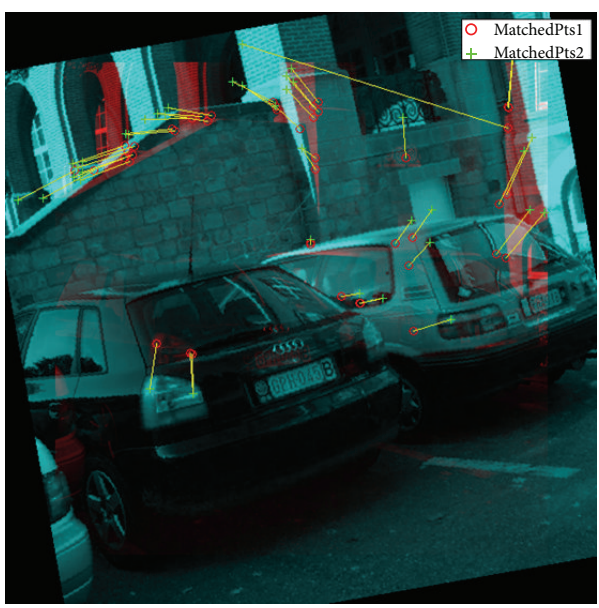

(c)

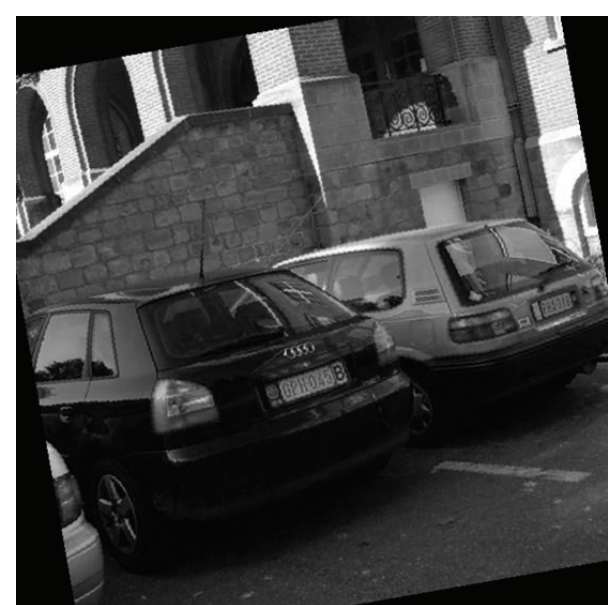

(b)

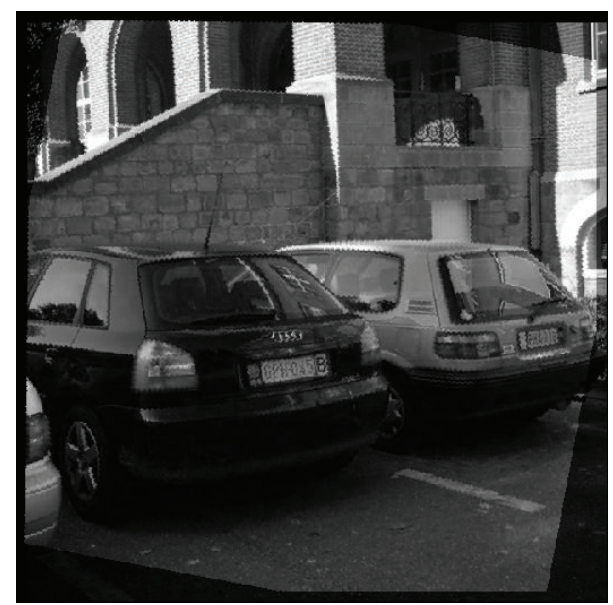

(d)

FIGURE 6: The images with different brightness to be registered.

TABLE 5: Comparison of this algorithm and the known parameters.

\begin{tabular}{lcc}
\hline & $\begin{array}{c}\text { The known } \\
\text { parameters }\end{array}$ & $\begin{array}{c}\text { Calculated } \\
\text { parameters }\end{array}$ \\
\hline The zoom & 1.2 & 1.2045 \\
\hline Rotation angle & 10 & 10 \\
\hline Translation vector & $(10,10)$ & $(9,10)$ \\
\hline Registration time & & $3.47 \mathrm{~s}$ \\
\hline
\end{tabular}

rotation angle influence the precision of translation parameters using the traditional phase correlation method. The two-step iterative method proposed in this paper, combining the SURF feature points extraction and phase correlation method, avoids the huge computation using the fast algorithm based on frequency domain. This paper, respectively, validates different types of visible light images with Matlab simulation. The results show that this algorithm ensures the registration precision, reduces the registration time, makes up for the defects of the traditional phase correlation method, and meets the requirement of high precision and fast image registration system.

\section{Conflict of Interests}

The authors declare that there is no conflict of interests regarding the publication of this paper.

\section{References}

[1] B. Zitová and J. Flusser, "Image registration methods: a survey," Image and Vision Computing, vol. 21, no. 11, pp. 977-1000, 2003.

[2] Y. S. Kim, J. H. Lee, and J. B. Ra, "Multi-sensor image registration based on intensity and edge orientation information," Pattern Recognition, vol. 41, no. 11, pp. 3356-3365, 2008.

[3] M. Brown and D. G. Lowe, "Automatic panoramic image stitching using invariant features," International Journal of Computer Vision, vol. 74, no. 1, pp. 59-73, 2007.

[4] X. Qu, F. Zhao, M. Zhou, and H. Huo, "A novel fast and robust binary affine invariant descriptor for image matching," Mathematical Problems in Engineering, vol. 2014, Article ID 129230, 7 pages, 2014. 
[5] K. Mikolajczyk and C. Schmid, "Scale \& affine invariant interest point detectors," International Journal of Computer Vision, vol. 60, no. 1, pp. 63-86, 2004.

[6] J. Bigot, F. Gamboa, and M. Vimond, "Estimation of translation, rotation, and scaling between noisy images using the FourierMellin transform," SIAM Journal on Imaging Sciences, vol. 2, no. 2, pp. 614-645, 2009.

[7] L. Lucchese and G. M. Cortelazzo, "A noise-robust frequency domain technique for estimating planar roto-translations," IEEE Transactions on Signal Processing, vol. 48, no. 6, pp. 17691786, 2000.

[8] Y. Keller, A. Averbuch, and O. Miller, "Robust phase correlation," in Proceedings of the 17th International Conference on Pattern Recognition, pp. 740-743, August 2004.

[9] J. Fan, L. Hu, and H. Ling, "An improved image registration method based on wavelet transform," Computer Engineering, vol. 36, no. 5, pp. 212-214, 2010.

[10] Z. Zheng and Z. Ye, "Image registration algorithm based on phase-correlation," Journal of Data Acquisition and Processing, vol. 21, no. 4, pp. 444-449, 2006.

[11] H. Foroosh, J. B. Zerubia, and M. Berthod, "Extension of phase correlation to subpixel registration," IEEE Transactions on Image Processing, vol. 11, no. 3, pp. 188-200, 2002.

[12] C. D. Kuglin and D. C. Hines, "The phase correlation image alignment method," in Proceedings of the IEEE International Conference on Cybernetics and Society, pp. 163-165, New York, NY, USA, 1975.

[13] Y. Keller, A. Averbuch, and M. Israeli, "Pseudopolar-based estimation of large translations, rotations, and scalings in images," IEEE Transactions on Image Processing, vol. 14, no. 1, pp. 12-22, 2005.

[14] X.-M. Li, X.-P. Zhao, L. Zheng, and Z.-Y. Hu, "Image registration technique based on Fourier-Mellin transform and its extended applications," Chinese Journal of Computers, vol. 29, no. 3, pp. 466-472, 2006.

[15] J. N. Sarvaiya, S. Patnaik, and S. Bombaywala, "Image registration using log-polar transform and phase correlation," in Proceedings of the IEEE 10th Region Conference, pp. 1-5, November 2009.

[16] H. Bay, A. Ess, T. Tuytelaars, and L. Van Gool, "Speeded-up robust features," Computer Vision and Image Understanding, vol. 110, no. 3, pp. 346-359, 2008.

[17] Y. Ke and R. Sukthankar, "PCA-SIFT: a more distinctive representation for local image descriptors," in Proceedings of the IEEE Computer Society Conference on Computer Vision and Pattern Recognition (CVPR '04), vol. 2, pp. II506-II513, July 2004. 


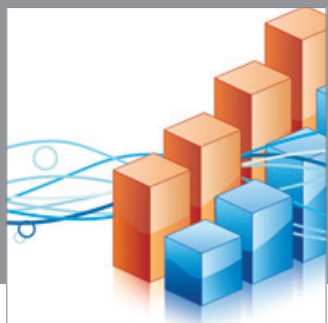

Advances in

Operations Research

mansans

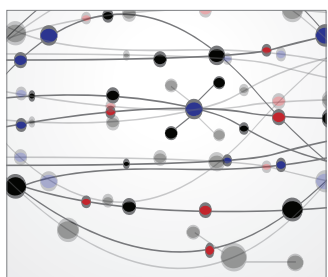

The Scientific World Journal
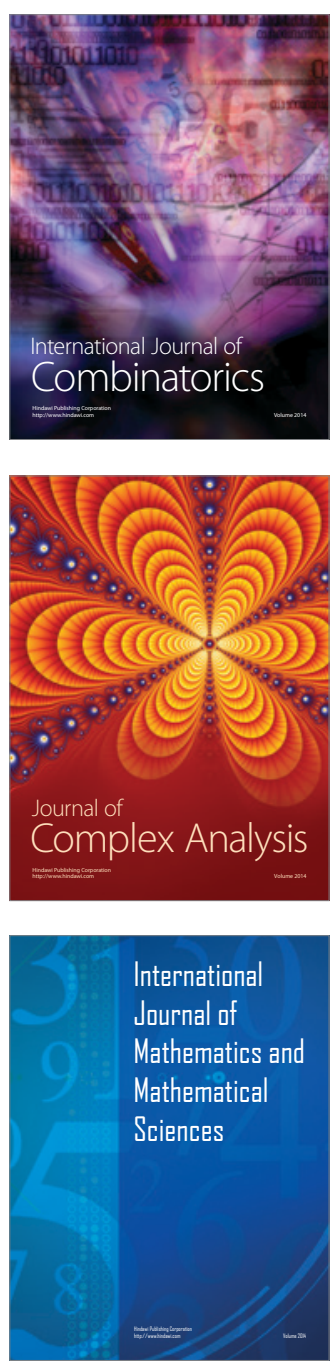
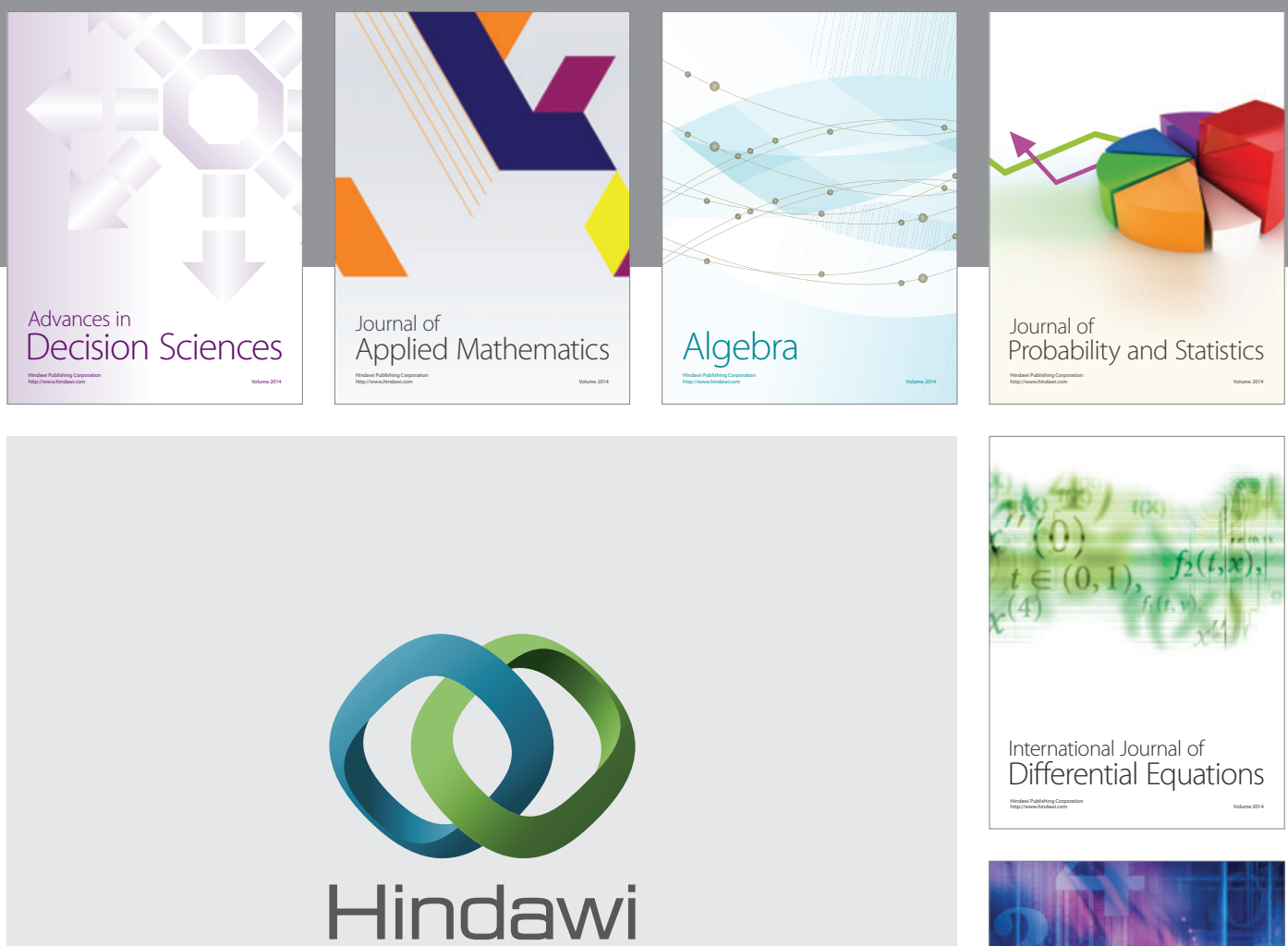

Submit your manuscripts at http://www.hindawi.com
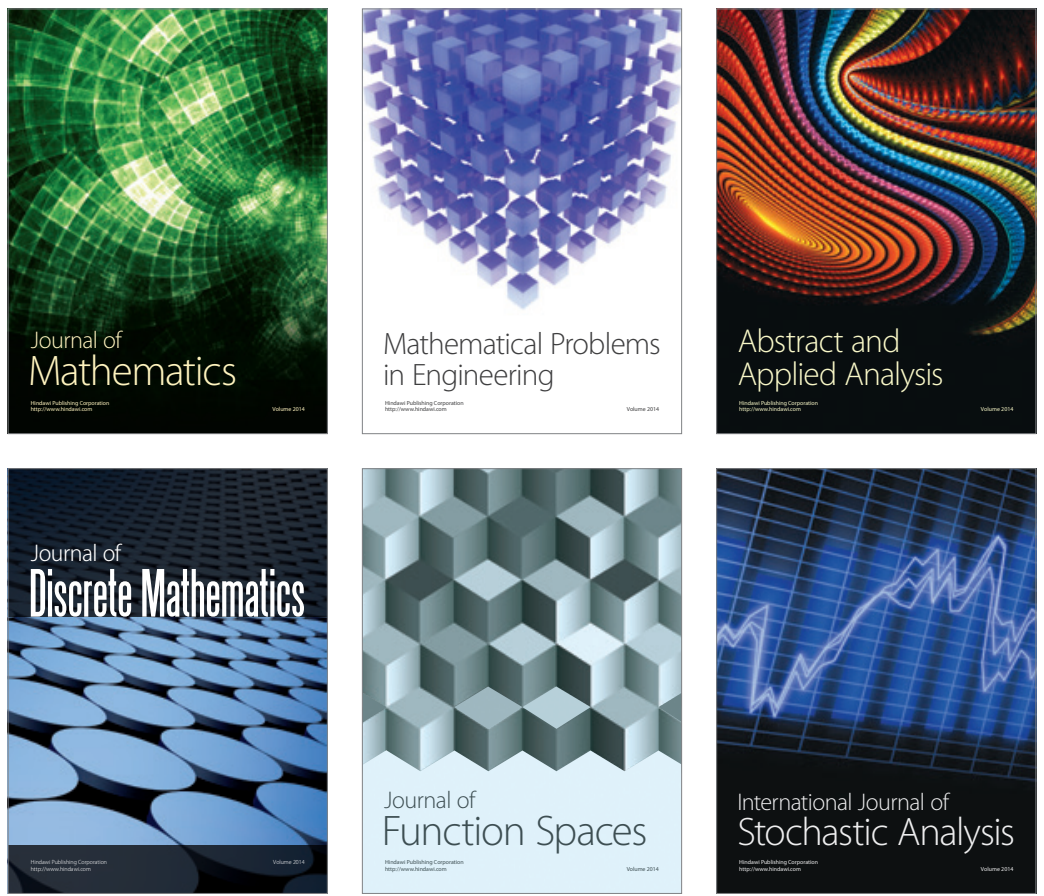

Journal of

Function Spaces

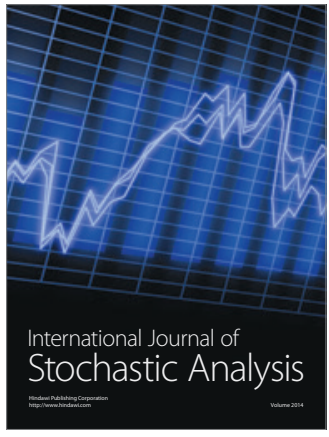

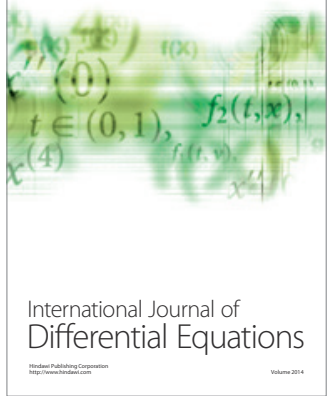
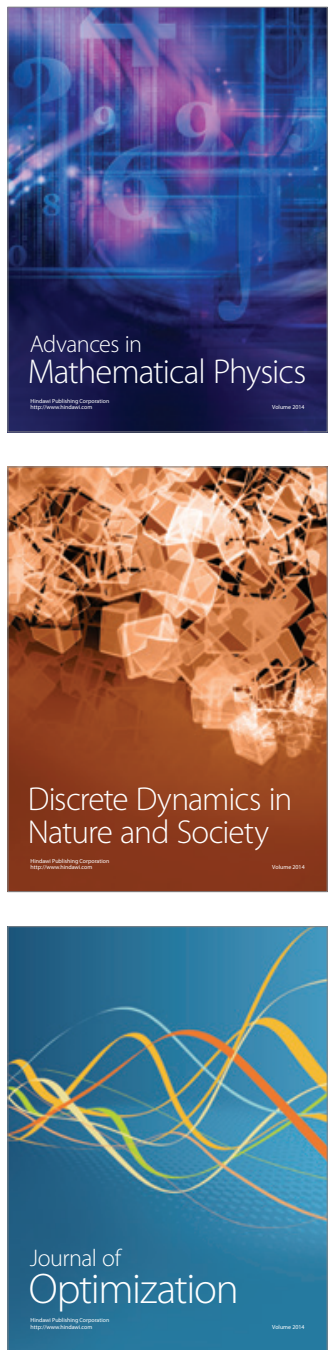